\title{
Video Article \\ Neurodegeneration in an Animal Model of Chronic Amyloid-beta Oligomer Infusion Is Counteracted by Antibody Treatment Infused with Osmotic Pumps
}

\author{
Ahmadali Sajadi ${ }^{1,3}$, Chloé Provost ${ }^{3}$, Brendon Pham ${ }^{2}$, Jonathan Brouillette ${ }^{1,3}$ \\ ${ }^{1}$ Department of Pharmacology, Université de Montréal \\ ${ }^{2}$ Department of Neuroscience, Université de Montréal \\ ${ }^{3}$ Hôpital du Sacré-coeur de Montréal Research Center, Université de Montréal
}

Correspondence to: Jonathan Brouillette at jonathan.brouillette@umontreal.ca

URL: https://www.jove.com/video/54215

DOI: doi: $10.3791 / 54215$

Keywords: Medicine, Issue 114, Amyloid-beta oligomers, osmotic pumps, Animal model, Intracerebral infusion, Neurodegeneration, Cannula, Neurobiology

Date Published: 8/14/2016

Citation: Sajadi, A., Provost, C., Pham, B., Brouillette, J. Neurodegeneration in an Animal Model of Chronic Amyloid-beta Oligomer Infusion Is Counteracted by Antibody Treatment Infused with Osmotic Pumps. J. Vis. Exp. (114), e54215, doi:10.3791/54215 (2016).

\section{Abstract}

Decline in hippocampal-dependent explicit memory (memory for facts and events) is one of the earliest clinical symptom of Alzheimer's disease (AD). It is well established that synapse loss and ensuing neurodegeneration are the best predictors for memory impairments in AD. Latest studies have emphasized the neurotoxic role of soluble amyloid-beta oligomers $(A \beta O)$ that begin to accumulate in the human brain approximately 10 to $15 \mathrm{yr}$ before the clinical symptoms become apparent. Many reports indicate that soluble Aßo correlate with memory deficits in AD models and humans. The Aßo-induced neurodegeneration observed in neuronal and brain slice cultures has been more challenging to reproduce in many animal models. The model of repeated $A \beta$ o infusions shown here overcome this issue and allow addressing two key domains for developing new disease modifying therapies: identify biological markers to diagnose early $A D$, and determine the molecular mechanisms underpinning $A \beta o$-induced memory deficits at the onset of $A D$. Since soluble $A \beta o$ aggregate relatively fast into insoluble $A \beta$ fibrils that correlate poorly with the clinical state of patients, soluble $A \beta 0$ are prepared freshly and injected once per day during six days to produce marked cell death in the hippocampus. We used cannula specially design for simultaneous infusions of A 30 and continuous infusion of $A \beta 0$ antibody (6E10) in the hippocampus using osmotic pumps. This innovative in vivo method can now be used in preclinical studies to validate the efficiency of new $A D$ therapies that might prevent the deposition and neurotoxicity of $A \beta O$ in pre-dementia patients.

\section{Video Link}

The video component of this article can be found at https://www.jove.com/video/54215/

\section{Introduction}

It was initially proposed that accumulation of insoluble $A \beta$ species in the brain was central to $A D$ pathogenesis. ${ }^{1,2}$ However, amyloid plaques are also detected in some cognitively normal elderly. ${ }^{3-7}$ To overcome the poor correlation existing between plaque depositions and cognitive deficits in $A D$, latest reports have shown the presence of toxic soluble $A \beta O$ at the onset of the disease, which correlate much better with the clinical state of the patients. ${ }^{8-14}$ Since the process of $A \beta$ oligomerization is very dynamic, it was suggested that neurotoxicity is induced by various $A \beta 0$ instead of only one specific type of oligomer. ${ }^{14-16}$ Since many studies have shown that Aßo can initiate synapse dysfunctions prior to synapse and neuronal loss, ${ }^{17-24}$ current theories indicate that A $\beta$-related treatment might be effective in early AD rather than at later stages as tested so far in clinical trials.

One hallmark feature of $A D$ pathogenesis is the massive and widespread cell death observed in the late stages of the disease, and the significant synapse and neuronal loss observed in localized brain regions when memory deficits become detectable at the clinical level. The perforant pathway that projects from the entorhinal cortex $(E C)$ to the dentate gyrus (DG) is perturbed markedly at the early onset of $A D{ }^{25,26}$ During the prodromal state of $A D$ when mild cognitive impairment $(\mathrm{MCl})$ becomes apparent significant cell death is detected in the EC as well as synaptic loss in the DG. ${ }^{25,26}$

Although a large body of evidence has pinpointed the toxic action of soluble $A \beta O$ in early $A D,{ }^{8-14} A \beta O-i n d u c e d$ neurodegeneration observed in neuronal culture or organotypic brain slice culture has been more challenging to reproduce in animal models. ${ }^{27}$ Most of the transgenic $A D$ models overexpressing $A \beta$ have amyloid plaques, tau hyperphosphorylation, synaptic deficiency and memory deficits. ${ }^{27}$ However, these models have been much less successful in modelling cell death observed in the hippocampus of AD patients. To overcome these technical issues we developed a model based on intracerebral infusions of soluble $A \beta O$. We reported earlier that repeated hippocampal infusions of soluble $A \beta 0$ induce gradual neuronal loss and tau hyperphosphorylation, two pathological hallmarks associated with memory decline in $A D{ }^{28} \mathrm{Here}$, we are showing a novel method to test $A D$ therapies using cannula specially design for simultaneous infusions of $A \beta 0$ and continuous infusion of $A \beta 0$ antibody (6E10) with osmotic pumps. 
Osmotic pumps provide a unique way to test in vivo the efficiency of any antibody (or other compounds) against Aßo-induced neurodegeneration directly at the infusion site of $A \beta$. Thus, these pumps represent a convenient tool to establish a solid proof-of-concept regarding the mechanisms of action of potential therapeutic agents in AD. Since recent reports point out the critical impact of soluble Aßo in the early stages of $A D$, many treatments directed toward $A \beta o$ are actually being tested by academic and pharmaceutical laboratories. This novel animal model allows mimicking the synaptic and neuronal loss observed in early AD, and osmotic pumps are used to infuse continuously treatment agents specifically at the $A \beta 0$ infusion site. The repetitive failures of $A D$ therapies tested over the last few years in mild to moderate patients as prompted researchers to initiate trials in pre-dementia patients before A $\beta$ o begin to accumulate abundantly and generate irreversible brain damage. In this context, testing new compounds that prevent the deposition and consequently the neurotoxicity of $A \beta 0$ might be of interest in pre-clinical patients.

\section{Protocol}

Ethics statement: The animal protocol for this project obtained the approval from the Animal Care Committee of the Hôpital du Sacré-Coeur de Montréal in compliance with the guidelines of the Canadian Council on Animal Care.

\section{Catheter Preparation before Stereotaxic Surgery}

1. Cut PE50 catheters ( $6 \mathrm{~cm}$ in length) that will be used to connect cannula with osmotic pumps (see Figure 1A). Fill PE50 catheters with artificial cerebrospinal fluid (aCSF) and seal both ends of the catheters. Keep the catheter at $4{ }^{\circ} \mathrm{C}$ until used.

\section{Cannula Implantation by Stereotaxy}

1. Perform the surgery in sterile conditions. Sterilize all the surgical instruments and materials by autoclaving. Clean the stereotaxic apparatus and the working area thoroughly, and disinfected with a $70 \%$ ethanol solution. Wear a surgical mask, hair bonnet and sterile gloves.

2. Anesthetize rats by injecting intraperitoneally (i.p.) a solution of ketamine and xylazine (100 mg/kg and $10 \mathrm{mg} / \mathrm{kg}$, respectively). Confirm anesthesia by checking movement after a gentle toe pinch.

3. Inject a non-steroidal anti-inflammatory drug (Meloxicam; $1 \mathrm{mg} / \mathrm{kg}$ ) subcutaneously (s.c.) to the anesthetized-animal at least $30 \mathrm{~min}$ prior to surgery.

4. Shave the head of the animal using clippers and disinfect the skin with a solution of chlorhexidine gluconate $2 \%$ and isopropyl alcohol $2 \%$ three times. Apply veterinary ophthalmic ointment on eyes to prevent dryness while under anesthesia.

5. Place the animal on a stereotaxic frame with the ear bars. Fix one cannula on the holder arm of the stereotaxic frame. Inject (s.c.) a local anesthetic agent (bupivacaine $(1.5 \mathrm{mg} / \mathrm{kg})$ and lidocaine $(1.5 \mathrm{mg} / \mathrm{kg})$ on the top of the head. Anesthesia is maintains during the whole procedure by placing a nose cone delivering $3 \%$ isoflurane. The entire surgical field should be draped off, but it was not done here to better demonstrate the technique.

6. Make an incision of $3 \mathrm{~cm}$ on the top of the head with a scalpel. Install 4 clamps around the incision to leave the skull clear. Using a round-tip scissor, make a pocket $\left(2 \times 2 \mathrm{~cm}^{2}\right)$ under the skin between shoulder blades of the animal.

7. Scrape the periosteum of the skull with a blade. Apply a gauze pad on the skull if bleeding.

8. Verify that the skull is flat and well aligned on the stereotaxic frame. To make sure that the skull is flat, check the height coordinates at the bregma and at the lambda. Take the coordinates of the bregma that will be used as the reference point.

9. Starting from the bregma, calculate the coordinates of the two guide cannula that will be implanted bilaterally in the hippocampus (anteroposterior: $-0.42 \mathrm{~cm}$; mediolateral: $\pm 0.30 \mathrm{~cm}$; dorsoventral: $-0.28 \mathrm{~cm}$ according to the Paxinos and Watson rat brain atlas) ${ }^{29}$. Indicate the position of the cannula with a marker.

10. Drill a hole $(0.5 \mathrm{~mm})$ in the skull at the implantation point of both cannula. Drill two other holes approximately $5 \mathrm{~mm}$ above and below those points to insert screws that will solidify cannula when applying dental cement.

11. Cut one end of the catheter previously filled with aCSF with a scalpel, and insert it in the angle arm of the cannula. Fix the first cannula with dental cement. Avoid putting dental cement around the position on the second cannula.

12. Let the dental cement dry for 2 - 3 min, then remove the holder arm from the first cannula, and fix the second cannula in it. Repeat steps 2.11 and 2.12. Put dummy cannula on both guide cannula. Ensure that the dummy and guide cannula are of the same length to prevent tissue infiltration and blocking the cannula.

13. Insert the free end of both catheters in the pocket previously made between shoulder blades of the animal. Remove the clamps and stitch the skin with a suture thread $4-0$.

NOTE: The top guide cannula needs to be accessible for upcoming $A \beta$ o infusions.

14. Remove the animal from the stereotaxic frame and put it back in its cage. During post-surgical recovery, place the cage on a heating water blanket until the animal wakes up. The cage should be partially on the pad so the rat can move away from the heat if needed. Monitor the animal constantly until it regains sufficient consciousness to maintain sternal recumbency.

15. Return rats to the animal facility to recover from the surgery for 10 days under close monitoring. NOTE: Do not return an animal that has undergone surgery to the company of other animals until fully recovered. Meloxicam (1 mg/kg) is given once per day during two days following surgery to treat pain.

\section{Osmotic Pump Installation}

1. One day before installation, fill osmotic pumps with $6 \mathrm{E} 10$ antibody $(1 \mathrm{mg} / \mathrm{ml} ; 100 \mu \mathrm{l})$ and control lgG1 antibody $(1 \mathrm{mg} / \mathrm{ml} ; 100 \mu \mathrm{l})$ according to the manufacturer's instructions under sterile condition. Keep the pumps in sterile distilled water at $37^{\circ} \mathrm{C}$ overnight to activate pumps.

2. Anesthetize rats with isoflurane $3 \%$ to install osmotic pumps $(0.5 \mu \mathrm{l} / \mathrm{hr}$ for 7 days). Shave between shoulder blades and disinfect the skin with a solution of chlorhexidine gluconate $2 \%$ and isopropyl alcohol $2 \%$. Make an incision of $2 \mathrm{~cm}$ with a scalpel between shoulder blades to locate the PE50 catheters connected to the guide cannula. 
3. Cut the end of the PE50 catheters containing dental cement with a scalpel. Connect the osmotic pumps to the PE50 catheters, and add some dental cement at the pump and catheter junction to secure the connection.

4. Stitch the skin tightly with a suture thread 4-0. Put the animal back in its cage on a heating water pad. Observe the animal wake up rapidly from an isoflurane anesthesia (about $5 \mathrm{~min}$ ).

NOTE: The surgery takes approximately 5 to $10 \mathrm{~min}$.

\section{Aßo Infusions in Awake and Freely Moving Rats}

1. Prepare the $A$ Bo solution $(0.2 \mu \mathrm{g} / \mu \mathrm{l})$ as previously reported. ${ }^{28,30}$ Allow $A \beta$ o to aggregate dynamically and spontaneously for $1 \mathrm{hr}$ at room temperature before infusion.

2. Install two $10 \mu \mathrm{l}$ syringes on an infusion pump. Fill the syringes with $5 \mu \mathrm{l}$ of sterile distilled water. Cut two PE50 catheters to about $60 \mathrm{~cm}$ in length with a scalpel. Fill both catheters with sterile distilled water using $1 \mathrm{ml}$ syringes and $21 \mathrm{G}$ needles.

3. Keep the $1 \mathrm{ml}$ syringes at one end of the catheter and connect the other end to the Hamilton syringes. Remove the $1 \mathrm{ml}$ syringes and check that there is no air bubbles within the catheters.

4. Insert internal cannula at the end of PE50 catheters. Using sterile distilled water, fill the internal cannula connected to PE50 catheters up to1 $\mu \mathrm{l}$ on Hamilton syringes. Make an air bubble by pulling back the pistons up to $2 \mu \mathrm{l}$.

5. Mix the $A \beta$ o solution by pipetting up and down using a tip of minimum of adherence. Avoid forming bubbles during mixing. Fill both internal cannula with $1.5 \mu \mathrm{l}$ of $\mathrm{A}$ ßo solution by pulling back the pistons up to $3.5 \mu \mathrm{l}$.

6. Make lines with a marker before and after the air bubble done in both catheters. This serves as a check point of ongoing infusion. For infusion, bring the cage near the infusion pump and remove its cover.

7. Place the rat in a snuggle and tightly scrape the arm of the snuggle around its neck to immobilize the head of the rat. Remove dummy cannula from the two guide cannula. Insert the internal cannula previously prepared in the guide cannula. Verify that they are fully inserted and well-fixed to the base of the guide cannula.

8. Release rat from the snuggle and put it back in its cage to limit contention stress. Monitor closely to ensure that the catheters do not twist together and the infusion is done properly.

9. Turn on the infusion pump. Inject $1 \mu \mathrm{l}$ of $A \beta 0$ solution at a rate of $0.1 \mu \mathrm{l} / \mathrm{min}(10 \mathrm{~min})$. During infusion, check that the syringe piston moves from $3.5 \mu \mathrm{l}$ to $2.5 \mu \mathrm{l}$, and that the air bubble in both PE50 catheters move continuously.

10. Leave the internal cannula in place for another 5 min after infusion to allow efficient diffusion of Aßo solution. Bring the rat back in the snuggle to remove internal cannula and capped guide cannula to prevent reflux of the injected solution. Use dummy cannula that stop just before the angle arm of the cannula. Return the animal in its cage.

11. Repeat $A \beta$ infusion once per day over 6 consecutive days. Euthanize the animal by decapitation.

\section{Representative Results}

The neurotoxic effect of $A \beta 0$ was investigated in Long-Evans rats by implanting cannula in the DG of the hippocampus. Soluble $A \beta 0$ were injected every day over six consecutive days. We used cannula specially design for simultaneous infusions of $A \beta$ and continuous infusion of $6 \mathrm{E} 10$ or control IgG1 antibody in the hippocampus using osmotic pumps (Figure 1A). For immunohistochemistry rats were anesthetized, perfused transcardially with $0.9 \% \mathrm{NaCl}$, and brains immersed in $4 \%$ paraformaldehyde solution for $48 \mathrm{hr}$ and $15 \%$ sucrose solution for $24 \mathrm{hr}$ Free-floating coronal sections $(40 \mu \mathrm{m})$ were treated with $0.3 \% \mathrm{H}_{2} \mathrm{O}_{2}$ for $30 \mathrm{~min}$, blocked in $1 \%$ goat serum for $1 \mathrm{hr}$, and incubated overnight at $4{ }^{\circ} \mathrm{C}$ with an anti-pan-A $\beta$ antibody. Sections were treated with $70 \%$ formic acid for 3 min before applying the antibody. Detection was performed using the $\mathrm{ABC}$ complex and a $0.05 \%$ 3,3-diaminobenzidine solution. Sections were mounted on gelatin-coated glass slides, air-dried $2 \mathrm{hr}$, dehydrated $(30,70,95$, and $100 \%$ alcohol), incubated in toluene $5 \mathrm{~min}$, and coverslipped. For cresyl violet staining, sections were incubated 10 min in a $0.5 \%$ cresyl violet solution, incubated $1 \mathrm{~min}$ in a $0.5 \%$ acetic acid solution, decolored $(70,95$, and $100 \%$ alcohol), immersed in toluene 5 $\mathrm{min}$, and covered with glass coverslip.

The results presented here show the deposition of $A \beta O$ in the DG in vicinity of the infusion site, and cell death associated with this accumulation. We found that the level A $\mathrm{B}$ o was substantially decreased by $6 \mathrm{E} 10$ antibody treatment (Figure 1B). Marked neurodegeneration was observed near the injecting site of $A \beta O$, and was attenuated by $6 E 10$ antibody treatment (Figure 1B). These results are consistent with $A \beta 0$ accumulation that we observed in the DG following repeated $A \beta$ o infusions in mice. ${ }^{28}$ Clearance of amyloid deposition by immunotherapy with $6 \mathrm{E} 10$ antibody shown here is in line with another report done in a transgenic AD mouse model. ${ }^{31}$ 


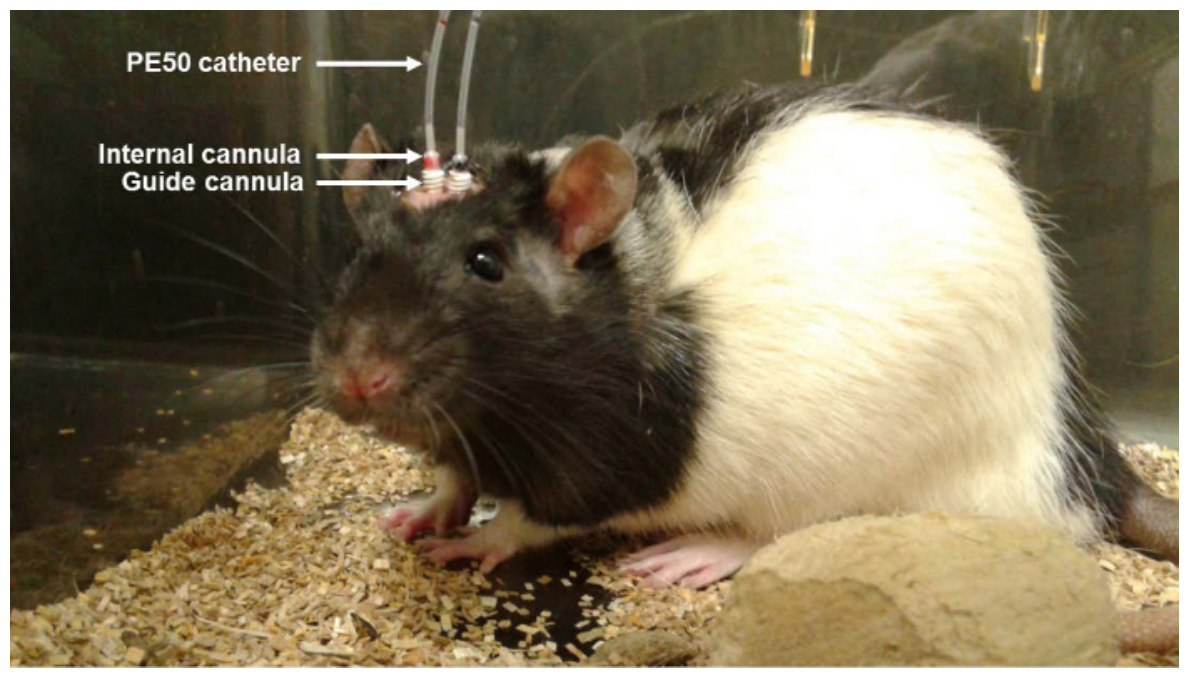

Figure 1. Photo of the Animal with Bilateral Cannula During Aßo Infusion. A solution of A $\beta 0(0.2 \mu g / \mu l ; 1 \mu \mathrm{l})$ was injected in awake and freely moving rats (once a day for 6 days) using PE50 catheters connected to internal cannula inserted into guide cannula. Treatment with control (Ctl) IgG1 or 6E10 antibody was infused directly at the site of Aßo infusion using osmotic pumps located subcutaneously between the shoulder blades of the animal. Please click here to view a larger version of this figure.

A

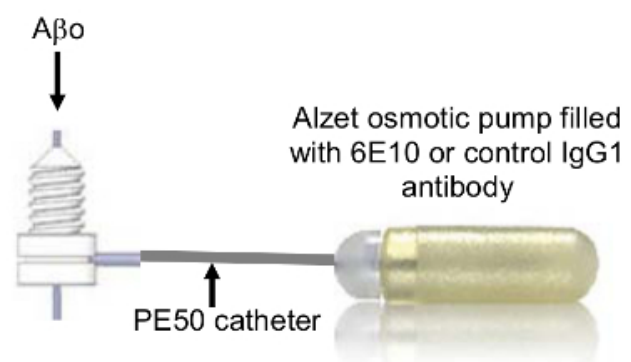

B

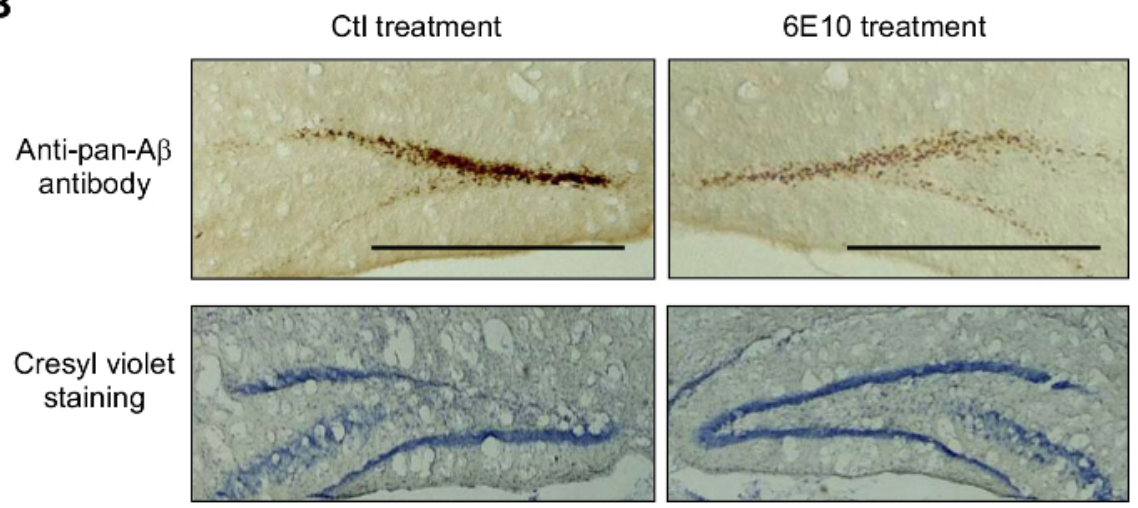

Figure 2. Neuronal Loss Induced by Aßo Deposition is Attenuated by 6 E10 Antibody Treatment. A, Aßo $(0.2 \mu \mathrm{g} / \mu \mathrm{l} ; 1 \mu \mathrm{l})$ was injected once a day during 6 consecutive days, and treated with $\mathrm{Ctl}$ ( $\operatorname{lgG} 1)$ or $6 \mathrm{E} 10$ antibody at the site of infusion using osmotic pumps. B, Representative accumulation of $A \beta O$ in the DG on a section immediately next to cannula insertion, and representative staining with cresyl violet showing cell loss. Scale bars: $50 \mu \mathrm{m}(\mathrm{n}=4)$ Please click here to view a larger version of this figure.

\section{Discussion}

There are critical steps within this protocol that required special attention. When implanting the cannula, avoid putting dental cement when it is too liquid to prevent blocking the hole of the second cannula. It is important to place dental cement at the free end of the P50 catheter attached to the pump to prevent irritation and a possible inflammatory response. The day of the stereotaxic surgery, use dummy cannula that are the same length as guide cannula to avoid blocking cannula. However, after installing pumps use shorter dummy cannula that stop before the angle arm of the cannula to allow proper infusion of the solution from the pump to the hippocampus. Monitor closely Aßo infusions and verify that the air bubble done in catheters is moving continuously during infusions. Always make sure that the injecting cannula is completely inserted into the guide cannula during infusions. 
If problem is encounter during $A \beta$ infusion, verify that the internal cannula is not blocked. If it is the case, flush sterile distilled water through the internal cannula. If the guide cannula is obstructed, turn the internal cannula into the guide cannula. Otherwise move the internal cannula up and down. Contention in the snuggle can be stressful for rats, especially on the first day. To decrease the stress of the animal, we recommend to manipulate and habituate rats to the snuggle before the stereotaxic surgery.

Many advantages can be attributed to this novel and flexible in vivo approach. Indeed, the nature of Aßo injected can be accurately control before infusion, and different type of $A \beta$ preparations (for example synthetic vs brain-derived $A \beta$ solutions) can be injected to evaluate their neurotoxicity in vivo. This model can also be used to investigate mechanisms by which various A $\beta$ species (e.g., monomers, low- and highmolecular-weight oligomers, protofibrils) can induce neurotoxic effects in vivo, and how treatments like immunotherapy might counteract their deleterious impact in the brain. Since the infusions are perform in awake, freely-moving animals, there are no confounding effects between anesthetic agents and the Aßo solution on signaling pathways, as shown in previous studies. ${ }^{32,33}$ Infusions in freely moving animal are also compatible with behavioral testing any time before and after the infusions.

Infusions of $A \beta O$ and pump installation can be done in animal of different ages to determine the effects of $A \beta 0$ and treatments during aging. Since neurodegeneration occurs in vicinity of the infusion site, synapse and neuronal loss can be induced in different and localized brain regions. The collateral infusion of $A \beta O$ and control (vehicle or scramble $A \beta$ ) allows controlling for any change within the same animal. Conversely, $A \beta 0$ or control solutions can be injected bilaterally in the right and left hippocampus, for example when testing animals in behavioral tasks. Infusion of $A \beta O$ and treatment can be done simultaneously or alternatively pumps can be installed after $A \beta O$ infusion to evaluate if the treatment is effective after $A \beta$ deposition. The same protocol described here can also be used when doing intracerebroventricular infusions of $A \beta 0$. The effect of $A \beta 0$ on intracellular signaling pathways can be evaluated before and after neuronal loss within a reasonably short time frame. The dose and number of $A \beta$ infusions can also be adjusted to obtain a more or less severe $A \beta$ pathogenicity.

Although very versatile, this technique has some limitations. Cannula implantation produces a mechanical disruption of the tissue and neuroinflammation in the first few days following surgery. Thus, it is essential to wait at least one week after surgery before starting $A \beta$ o infusion, and to add proper controls (injection of vehicle or inactive scramble $A \beta$ ) to take into account these events. Also, only a small volume of $A \beta 0$ can be infused to limit diffusion of the solution.

The osmotic pumps represent a convenient and unique delivery method for preclinical validation of agents designed to prevent $A \beta$-induced neurodegeneration. Since immunotherapy with the $6 \mathrm{E} 10$ antibody has been shown previously to decrease $A \beta$ accumulation in the brain, ${ }^{31}$ we used the 6E10 antibody as a proof-of-concept to validate our new in vivo approach. The used of osmotic pumps in this model might now be used to develop novel disease modifying therapies that could prevent the deposition and neurotoxicity of $A \beta O$ in pre-clinical AD patients.

\section{Disclosures}

Publication of this video-article is sponsored by ALZET Osmotic Pumps.

\section{Acknowledgements}

We thank Caroline Bouchard from the animal facility for the rat work. A.S. holds a J.A. De Sève master fellowship, and B.P. a COPSE fellowship from the Université de Montréal This work was funded by grants attributed to J.B. from FRQS-Pfizer and start-up funds from Hôpital du SacréCoeur de Montréal Research Center.

\section{References}

1. Hardy, J. A., \& Higgins, G. A. Alzheimer's disease: the amyloid cascade hypothesis. Science. 256, 184-185 (1992)

2. Selkoe, D. J. Toward a comprehensive theory for Alzheimer's disease. Hypothesis: Alzheimer's disease is caused by the cerebral accumulation and cytotoxicity of amyloid beta-protein. Ann. N.Y. Acad. Sci. 924, 17-25 (2000).

3. Terry, R. D. et al. Physical basis of cognitive alterations in Alzheimer's disease: synapse loss is the major correlate of cognitive impairment. Ann. Neurol. 30, 572-580 (1991).

4. Giannakopoulos, P. et al. Tangle and neuron numbers, but not amyloid load, predict cognitive status in Alzheimer's disease. Neurology 60 , 1495-1500 (2003).

5. Price, J. L., \& Morris, J. C. Tangles and plaques in nondemented aging and "preclinical" Alzheimer's disease. Ann. Neurol. 45, 358-368 (1999).

6. Reiman, E. M. et al. Fibrillar amyloid-beta burden in cognitively normal people at 3 levels of genetic risk for Alzheimer's disease. Proc. Natl. Acad. Sci. USA 106, 6820-6825 (2009).

7. Aizenstein, H. J. et al. Frequent amyloid deposition without significant cognitive impairment among the elderly. Arch. Neurol. 65, 1509-1517 (2008).

8. Mc Donald, J. M. et al. The presence of sodium dodecyl sulphate-stable Abeta dimers is strongly associated with Alzheimer-type dementia. Brain 133, 1328-1341 (2010).

9. Tomic, J. L., Pensalfini, A., Head, E., \& Glabe, C. G. Soluble fibrillar oligomer levels are elevated in Alzheimer's disease brain and correlate with cognitive dysfunction. Neurobiol. Dis. 35, 352-358 (2009).

10. Naslund, J. et al. Correlation between elevated levels of amyloid beta-peptide in the brain and cognitive decline. JAMA 283, 1571-1577 (2000).

11. Hardy, J. Amyloid double trouble. Nat. Genet. 38, 11-12 (2006).

12. Hardy, J., \& Selkoe, D. J. The amyloid hypothesis of Alzheimer's disease: progress and problems on the road to therapeutics. Science. 297, 353-356 (2002).

13. Haass, C., \& Selkoe, D. J. Soluble protein oligomers in neurodegeneration: lessons from the Alzheimer's amyloid beta-peptide. Nat. Rev. Mol. Cell Biol. 8, 101-112 (2007) 
14. McLean, C. A. et al. Soluble pool of Abeta amyloid as a determinant of severity of neurodegeneration in Alzheimer's disease. Ann. Neurol. 46, 860-866 (1999).

15. Hepler, R. W. et al. Solution state characterization of amyloid beta-derived diffusible ligands. Biochemistry 45, 15157-15167 (2006).

16. Martins, I. C. et al. Lipids revert inert Abeta amyloid fibrils to neurotoxic protofibrils that affect learning in mice. EMBO J. 27, 224-233 (2008).

17. Lesne, S. et al. A specific amyloid-beta protein assembly in the brain impairs memory. Nature 440, 352-357 (2006).

18. Hung, L. W. et al. Amyloid-beta peptide (Abeta) neurotoxicity is modulated by the rate of peptide aggregation: Abeta dimers and trimers correlate with neurotoxicity. J. Neurosci. 28, 11950-11958 (2008).

19. Ono, K., Condron, M. M., \& Teplow, D. B. Structure-neurotoxicity relationships of amyloid beta-protein oligomers. Proc. Natl. Acad. Sci. USA. 106, 14745-14750 (2009).

20. Lambert, M. P. et al. Diffusible, nonfibrillar ligands derived from Abeta1-42 are potent central nervous system neurotoxins. Proc. Natl. Acad. Sci. USA 95, 6448-6453 (1998).

21. Shankar, G. M. et al. Amyloid-beta protein dimers isolated directly from Alzheimer's brains impair synaptic plasticity and memory. Nat. Med. 14, 837-842 (2008).

22. Shankar, G. M. et al. Natural oligomers of the Alzheimer amyloid-beta protein induce reversible synapse loss by modulating an NMDA-type glutamate receptor-dependent signaling pathway. J. Neurosci. 27, 2866-2875 (2007).

23. Cleary, J. P. et al. Natural oligomers of the amyloid-beta protein specifically disrupt cognitive function. Nat. Neurosci. 8, 79-84 (2005).

24. Townsend, M., Shankar, G. M., Mehta, T., Walsh, D. M., \& Selkoe, D. J. Effects of secreted oligomers of amyloid beta-protein on hippocampal synaptic plasticity: a potent role for trimers. J. Physiol. 572, 477-492 (2006).

25. Gomez-Isla, T. et al. Profound loss of layer Il entorhinal cortex neurons occurs in very mild Alzheimer's disease. J. Neurosci. 16, 4491-4500 (1996).

26. DeKosky, S. T., Scheff, S. W., \& Styren, S. D. Structural correlates of cognition in dementia: quantification and assessment of synapse change. Neurodegeneration. 5, 417-421 (1996).

27. Brouillette, J. The Effects of Soluble Abeta Oligomers on Neurodegeneration in Alzheimer's Disease. Curr. Pharm. Des. 20, 2506-2519 (2014).

28. Brouillette, J. et al. Neurotoxicity and memory deficits induced by soluble low-molecular-weight amyloid-beta1-42 oligomers are revealed in vivo by using a novel animal model. J. Neurosci. 32, 7852-7861 (2012).

29. Paxinos, G., \& Watson, C. The rat brain in stereotaxic coordinates. New York: Academic Press. Fourth edition (1998).

30. Broersen, K. et al. A standardized and biocompatible preparation of aggregate-free amyloid beta peptide for biophysical and biological studies of Alzheimer's disease. PEDS 24, 743-750 (2011).

31. Thakker, D. R. et al. Intracerebroventricular amyloid-beta antibodies reduce cerebral amyloid angiopathy and associated micro-hemorrhages in aged Tg2576 mice. Proc. Natl. Acad. Sci. USA 106, 4501-4506 (2009).

32. Papon, M. A., Whittington, R. A., El-Khoury, N. B., \& Planel, E. Alzheimer's disease and anesthesia. Front. Neurosci. 4, 272 (2011).

33. Le Freche, H. et al. Tau phosphorylation and sevoflurane anesthesia: an association to postoperative cognitive impairment. Anesthesiology 116, 779-787 (2012). 